University of Wollongong

Research Online

Australian Institute for Innovative Materials -

Papers

Australian Institute for Innovative Materials

$1-1-2014$

Direct evidence of Ni magnetic moment in TbNi2Mn-X-ray magnetic circular dichroism

D H. Yu

ANSTO

Meng-Jie Huang

National Synchrotron Radiation Research Centre

Jianli Wang

University of Wollongong, jianli@uow.edu.au

Hui-Chia Su

National Synchrotron Radiation Research Centre

Hong-Ji Lin

National Synchrotron Radiation Research Center Taiwan

See next page for additional authors

Follow this and additional works at: https://ro.uow.edu.au/aiimpapers

Part of the Engineering Commons, and the Physical Sciences and Mathematics Commons

Research Online is the open access institutional repository for the University of Wollongong. For further information contact the UOW Library: research-pubs@uow.edu.au 


\title{
Direct evidence of Ni magnetic moment in TbNi2Mn-X-ray magnetic circular dichroism
}

\author{
Abstract \\ We have investigated the individual magnetic moments of $\mathrm{Ni}, \mathrm{Mn}$ and $\mathrm{Tb}$ atoms in the intermetallic \\ compound TbNi2Mn in the Laves phase (magnetic phase transition temperature TC $\sim 131 \mathrm{~K}$ ) by $\mathrm{X}$-ray \\ magnetic circular dichroism (XMCD) studies at $300 \mathrm{~K}, 80 \mathrm{~K}$ and $20 \mathrm{~K}$. Analyses of the experimental results \\ reveal that $\mathrm{Ni}$ atoms at $20 \mathrm{~K}$ in an applied magnetic field of $1 \mathrm{~T}$ carry an intrinsic magnetic moment of spin \\ and orbital magnetic moment contributions $0.53 \pm 0.01 \mu \mathrm{B}$ and $0.05 \pm 0.01 \mu \mathrm{B}$, respectively. These moment \\ values are similar to those of the maximum saturated moment of $\mathrm{Ni}$ element. A very small magnetic \\ moment of order

\section{Keywords} \\ dichroism, direct, evidence, ni, magnetic, moment, tbni2mn, x, ray, circular \\ Disciplines \\ Engineering | Physical Sciences and Mathematics

\section{Publication Details} \\ Yu, D., Huang, M., Wang, J. L., Su, H., Lin, H., Chen, C. \& Campbell, S. J. (2014). Direct evidence of Ni \\ magnetic moment in TbNi2Mn-X-ray magnetic circular dichroism. Journal of Magnetism and Magnetic \\ Materials, 370 32-36.

\section{Authors} \\ D H. Yu, Meng-Jie Huang, Jianli Wang, Hui-Chia Su, Hong-Ji Lin, Chien-Te Chen, and S J. Campbell
}




\title{
Direct evidence of Ni magnetic moment in $\mathrm{TbNi}_{2} \mathrm{Mn}$ - \\ X-ray Magnetic Circular Dichroism
}

D. H. Yu ${ }^{1 *}$, Meng-Jie Huang ${ }^{3}$, J. L. Wang ${ }^{1,2,4}$, Hui-Chia Su ${ }^{3}$, Hong-Ji Lin ${ }^{3}$, Chien-Te Chen ${ }^{3}$ and S. J. Campbell ${ }^{2}$

${ }^{1}$ Bragg Institute, Australian Nuclear Science and Technology Organisation, Lucas Heights, NSW 2234, Australia

${ }^{2}$ School of Physical, Environmental and Mathematical Sciences, University of New South Wales, Canberra at the Australian Defence Force Academy, ACT 2600, Australia

${ }^{3}$ National Synchrotron Radiation Research Center, 101 Hsin-Ann Road, Hsinchu Science Park, Hsinchu 30076, Taiwan

${ }^{4}$ Institute for Superconductivity and Electronic Materials, University of Wollongong, Wollongong, NSW 2522, Australia

\begin{abstract}
We have investigated the individual magnetic moments of $\mathrm{Ni}, \mathrm{Mn}$ and $\mathrm{Tb}$ atoms in the intermetallic compound $\mathrm{TbNi}_{2} \mathrm{Mn}$ in the Laves phase (magnetic phase transition temperature $\mathrm{T}_{\mathrm{C}} \sim 131 \mathrm{~K}$ ) by $\mathrm{x}$ ray magnetic circular dichroism (XMCD) studies at $300 \mathrm{~K}, 80 \mathrm{~K}$ and $20 \mathrm{~K}$. Analyses of the experimental results reveal that $\mathrm{Ni}$ atoms at $20 \mathrm{~K}$ in an applied magnetic field of $1 \mathrm{~T}$ carry an intrinsic magnetic moment of spin and orbital magnetic moment contributions $0.53 \pm 0.01 \mu_{\mathrm{B}}$ and $0.05 \pm 0.01 \mu_{\mathrm{B}}$, respectively. These moment values are similar to those of the maximum saturated moment of Ni element. A very small magnetic moment of order $<0.1 \mu_{\mathrm{B}}$ has been measured for Mn. This suggests that $\mathrm{Mn}$ is antiferromagnetically ordered across the two nearly equally occupied sites of $16 \mathrm{~d}$ and $8 \mathrm{a}$. A magnetic moment of up to $\sim 0.3 \mu_{\mathrm{B}}$ has been observed for the $\mathrm{Tb}$ atoms. Identification of a magnetic moment on the $\mathrm{Ni}$ atoms has provided further evidence for the mechanism of enhancement of the magnetic phase transition temperature in $\mathrm{TbNi}_{2} \mathrm{Mn}$ compared
\end{abstract}


with $\mathrm{TbNi}_{2}\left(\mathrm{~T}_{\mathrm{C}} \sim 37.5 \mathrm{~K}\right)$ and $\mathrm{TbMn}_{2}\left(\mathrm{~T}_{\mathrm{C}} \sim 54 \mathrm{~K}\right)$ due to rare earth-transition metal $(\mathrm{R}-\mathrm{T})$ and transition metal - transition metal (T-T) interactions. The behaviour of the $\mathrm{x}$-ray magnetic circular dichroism spectra of $\mathrm{TbNi}_{2} \mathrm{Mn}$ at $300 \mathrm{~K}, 80 \mathrm{~K}$ and $20 \mathrm{~K}$ - above and below the magnetic ordering temperature $\mathrm{T}_{\mathrm{C}} \sim 131 \mathrm{~K}$ - is discussed.

Keywords: Intermetallic, XMCD, Magnetic, $\mathrm{TbNi}_{2} \mathrm{Mn}$ 


\section{Introduction}

Transition-metal (TM) and rare-earth (RE) magnetic compounds continue to be a subject of interesting and extensive investigations as they have been for many decades. ${ }^{1,2}$ The competition among the interactions of rare earth elements (R-R), rare earth and transition metal (R-T) and transition metal elements (T-T) can trigger new phenomena; this in turn leads to the prospect of new technological applications such as the magnetocaloric effect ${ }^{3-6}$ and giant magnetostriction properties. ${ }^{7}$ One of the well-established fundamental models is the disappearance of the magnetic moment of nickel in $\mathrm{RE}-\mathrm{Ni}_{2}$ compounds as a result of the filling up of the $\mathrm{Ni} 3 \mathrm{~d}$ band by the outershell electrons of the RE elements. ${ }^{8-10}$ This paradigm, supported by neutron diffraction and band calculations, has generally been accepted in the magnetism research society until the observation of a Ni magnetic moment in $\mathrm{GdNi}_{2}$ by Mizumaki et al. (2003) ${ }^{11}$ and Yano et al. (2006) ${ }^{12}$ using x-ray magnetic circular dichroism and magnetic Compton profile methods, respectively. Occurrence of a Ni magnetic moment will enhance the R-T and T-T interactions and consequently may lead to new phenomena.

The first report of a new class of TM-RE magnetic compound $\mathrm{RNi}_{2} \mathrm{Mn}(\mathrm{R}=\mathrm{Tb}, \mathrm{Dy}$, Ho, and Er) ${ }^{13}$ has attracted great interest in the study of the magnetic structure and properties of these new magnetic compounds. This class of materials normally crystallizes in the $\mathrm{MgCu}_{2}$-type Laves phase (C15 space group Fd-3m). ${ }^{13}$ However, a different phase of structure (C15b space group F-43m) has also been reported for $\mathrm{TbNi}_{2} \mathrm{Mn}$ by Mushnikov et al. (2009). ${ }^{14}$ Among this group of materials (including $\mathrm{TbNi}_{2}$ and $\mathrm{TbMn}_{2}$ ), $\mathrm{TbNi}_{2} \mathrm{Mn}$ has been shown to exhibit the largest magnetic ordering temperature $\left(\mathrm{T}_{\mathrm{C}} \sim 131 \mathrm{~K}\right.$ to $\mathrm{T}_{\mathrm{C}} \sim 151 \mathrm{~K}$ ) and to exhibit a second order magnetic phase transition. ${ }^{15}$ The high magnetic transition temperature observed in this class of materials does not follow de Gennes rule alone and indicates possible contributions from R-T and/or T-T interactions due to nonzero magnetic moment from $\mathrm{Ni}$ and $\mathrm{Mn}$ transition elements. 
Magnetic moment values of $\mu^{\mathrm{Mn}}=1.4 \mu_{\mathrm{B}}$ for $\mathrm{Mn}$ and $\mu^{\mathrm{Ni}}=0.3 \mu_{\mathrm{B}}$ for Ni have been derived from neutron powder diffraction on an $\mathrm{AuBe}_{5}$-type $\mathrm{TbNi}_{2} \mathrm{Mn}$ sample; the moment for the $\mathrm{Ni}$ ion was determined by restricting the Tb moment below the free-ion value. ${ }^{14}$ Given this evidence for a non-zero value for the $\mathrm{Ni}$ magnetic moment in $\mathrm{TbNi}_{2} \mathrm{Mn}$, direct detection of a $\mathrm{Ni}$ magnetic moment is none the less required to clarify understanding of the mechanism for the high magnetic ordering temperature found for the new class of materials $\mathrm{RNi}_{2} \mathrm{Mn}$. Here, we report the findings of our studies of the behaviour of the individual magnetic moments of $\mathrm{Ni}, \mathrm{Mn}$ and $\mathrm{Tb}$ for the $\mathrm{TbNi}_{2} \mathrm{Mn}$ compound as determined by x-ray magnetic circular dichroism (XMCD), with particular interest in identifying the behaviour of a non-zero Ni magnetic moment in $\mathrm{RNi}_{2} \mathrm{Mn}$.

\section{Experiment}

The $\mathrm{TbNi}_{2} \mathrm{Mn}$ alloy was prepared by argon arc-melting the starting elements with purities of at least $99.9 \%$. The constituents were melted in the stoichiometric ratio of 1:2:1, plus an excess of Mn (less than 5\%) to compensate the Mn evaporation during the arc-melting and the subsequent annealing processes. The structure was characterised by x-ray powder diffraction (XRD) on a Rigaku Rint-2400 diffractometer with $\mathrm{Cu} K_{\alpha}$ radiation of wavelength $1.5418 \AA$ and the single phase nature of the sample with its $\mathrm{MgCu}_{2}$-type structure confirmed. ${ }^{13}$ The chemical compositions of the $\mathrm{TbNi}_{2} \mathrm{Mn}$ sample were determined by energy dispersive spectroscopy (EDS) and found to be $\mathrm{Tb}$ 25.3 $\pm 0.6 \%$, Ni $51.2 \pm 0.6 \%$, and Mn 24.6 $\pm 0.6 \%$, thus confirming the 1:2:1 stoichiometry within the experimental error. ${ }^{13}$ The temperature dependence of the magnetization as well as the hysteresis loops were measured in a superconducting quantum interference device (SQUID) magnetometer and in an extraction-sample magnetometer. Detailed structural information and magnetic properties of the $\mathrm{TbNi}_{2} \mathrm{Mn}$ sample, which has a magnetic ordering temperature of $\mathrm{T}_{\mathrm{C}}=131 \mathrm{~K}$, are provided in reference. $^{13}$ 
The XMCD experiment was conducted at the BL11A Dragon beam line at the National Synchrotron Radiation Research Centre in Taiwan. ${ }^{16}$ Measurements were performed at three sample temperatures of $\mathrm{T}=20 \mathrm{~K}, 80 \mathrm{~K}, 300 \mathrm{~K}$ with an applied magnetic field of $\mathrm{B}=1 \mathrm{~T}$. The size of the rectangularly shaped polycrystalline sample is $\sim 2 \mathrm{x} 2 \mathrm{x} 5 \mathrm{~mm}^{3}$. One of the surfaces parallel to the longer dimension was polished with superfine waterproof P1200 sandpaper followed by ultrasound cleaning in acetone first and then methanol each for about 10 minutes. The sample is then installed inside an ultra-high vacuum chamber immediately after the cleaning procedure and followed by pumping down the vacuum chamber within 20 minutes.

The external magnetic field was applied along the longer direction (labelled the y-axis) and in the polished surface plane. The propagation direction of the incident circular polarized light (about $85 \%$ polarisation) was $30^{\circ}$ with respect to the magnetic field direction. The total electron yield mode was used for the measurements of x-ray absorption spectra (XAS). The x-ray magnetic circular dichroism spectra were derived from the difference of the spin-resolved XAS with the magnetic field direction either parallel or antiparallel to the y-axis in the sample plane after background subtraction. ${ }^{17}$ The net orbital and spin moments were derived using the well-known sum rules for $\mathrm{x}$-ray magnetic circular dichroism. ${ }^{18,19}$ For Ni and Mn, the sum rule corresponding to the $\mathrm{L}_{2,3}$ edge was used with the $\langle\mathrm{Tz}>/<\mathrm{Sz}>$ term being neglected as the expectation value of the magnetic dipole operator $\langle\mathrm{Tz}>$ is usually very small with respect to the expectation value of the spin operator $<$ Sz $>$ for $3 d$ transition metals. ${ }^{19}$ The effective numbers of $3 d$ holes $n_{h}{ }^{N i}=1.59$ and $n_{h}{ }^{\text {Mn }}$ $=4.5$ for $\mathrm{Ni}$ and Mn, respectively, were taken based on band structure calculations. ${ }^{20,21}$ For Tb, the sum rule corresponding to the $\mathrm{M}_{4,5}$ edge was used with $<\mathrm{Tz}>/<\mathrm{Sz}>=-0.08$ and $\mathrm{n}_{\mathrm{h}}{ }^{\mathrm{Tb}}=6$ from band structure calculations. $^{22}$ The estimated uncertainty of the derived magnetic moment values is about $0.01 \mu_{\mathrm{B}}$; this is due primarily to the errors in light polarization and background subtraction. The potential for a signal due to oxidation of the top one or two monolayers of the sample could not be 
excluded. However the measurement should mainly probe the bulk properties of the alloy as the probe depth of the total electron yield is in the order of $10 \mathrm{~nm}$.

\section{Results and Discussions}

The Ni, Mn and Tb isotropic x-ray absorption spectra and x-ray magnetic circular dichroism spectra for $\mathrm{TbNi}_{2} \mathrm{Mn}$ at $20 \mathrm{~K}$ are presented in Figures 1, 2 and 3 respectively. Multiple structures have been observed as marked by capital letters from A to E in the XAS spectra. For the three elements, three distinct peaks appear at the $L_{3}\left(M_{5}\right)$ edges, while only two peaks can be identified at $L_{2}\left(M_{4}\right)$ edges.

Figure 1 (a) X-ray absorption spectra of the $\mathrm{Ni} L_{23}(2 p \rightarrow 3 d)$ of $\mathrm{TbNi}_{2} \mathrm{Mn}(\mathrm{T}=20 \mathrm{~K})$ and (b) X-ray magnetic circular dichroism of the $\mathrm{Ni} L_{23}(2 p \rightarrow 3 d)$ of $\mathrm{TbNi}_{2} \mathrm{Mn}(\mathrm{T}=20 \mathrm{~K} ; \mathrm{B}=1 \mathrm{~T})$. The peaks labelled A, B, C, D, E agree well with theoretical calculations as discussed in the text.

The observed Ni 2p XAS and XMCD spectra as shown in Figure 1 are generally consistent with the existing atomic multiplet calculations in a crystal field with local octahedral symmetry for free $\mathrm{Ni}^{2+}$ ion with initial $3 d^{8}$ and final $2 p 3 d^{9}$ configurations. ${ }^{23,24}$ Coulomb, spin-orbit, exchange interactions and interatomic screening and states mixing effects were included in the calculations for both initial and final states. The calculated spectra with crystal field strengths larger than $1 \mathrm{eV}$ approached the experimental observations for $\mathrm{TbNi}_{2} \mathrm{Mn}$. The $17 \mathrm{eV}$ splitting between the peaks $\mathrm{A}$ and D corresponds to the $L_{2}$ and $L_{3}$ separation due to the large core-hole spin-orbit interactions. ${ }^{23}$ The splitting between peaks $\mathrm{A}$ and $\mathrm{B}$ is a measure of the $2 p$ hole and $3 d$ electron exchange interaction. ${ }^{24}$ The measured splitting between peaks $\mathrm{A}$ and $\mathrm{B}$ in the $\mathrm{TbNi}_{2} \mathrm{Mn}$ compound is $1.75 \pm$ $0.06 \mathrm{eV}$ which is less than the values of $2.0 \mathrm{eV}$ and $1.9 \mathrm{eV}$ observed for trevorite and $\mathrm{NiO}$ respectively, ${ }^{24}$ but very close to the value of $1.78 \mathrm{eV}$ from $\mathrm{Pd}_{40} \mathrm{Ni}_{22.5} \mathrm{Fe}_{17.5} \mathrm{P}_{20}{ }^{25}$ The peak $\mathrm{C}$ is attributed to a $2 p_{3 / 2} 3 d^{10}$ final state configuration. ${ }^{26}$ At the $L_{2}$ edge, only two peaks - D and E - can be identified. A contribution from the $2 p_{3 / 2} 3 d^{10}$ final state configuration has not shown up possibly due 
to lifetime broadening effects. ${ }^{11}$ For the isotropic branching ratios $\mathrm{B}(\mathrm{i})$, defined as the integrated intensity of the $\mathrm{L}_{3}$ edge normalised to the integrated intensity of the whole XAS, ${ }^{23}$ our measured value is $\mathrm{B}(\mathrm{i})^{\text {meas }}=0.80$ which is in reasonable agreement with the calculated value of $\mathrm{B}(\mathrm{i})=0.75$ corresponding to the high-spin ground state with $1 \mathrm{eV}$ crystal field strength. ${ }^{23}$

While these multiple structures also appear in the XMCD spectrum for Ni electrons in $\mathrm{TbNi}_{2} \mathrm{Mn}$ (Figure 1(b)), the shape of the XMCD spectrum is found to be very similar to that measured on a Ni(111) single crystal. ${ }^{27}$ The present derived spin and orbital magnetic moment using XMCD sum rules are $0.53 \pm 0.01 \mu_{\mathrm{B}}$ and $0.05 \pm 0.01 \mu_{\mathrm{B}}$, respectively at $20 \mathrm{~K}$. These values agree well with the Ni single crystal results of $0.52 \mu_{\mathrm{B}}$ and $0.05 \mu_{\mathrm{B}}{ }^{27}$ This good agreement indicates that $\mathrm{Ni}$ in $\mathrm{TbNi}_{2} \mathrm{Mn}$ carries an intrinsic magnetic moment that is determined by the localised $3 d$ electrons as also evidenced by the match of the observed XAS with that from the atomic multiplet calculations. The fact that $\mathrm{Ni}$ atoms carry larger magnetic moments in $\mathrm{TbNi}_{2} \mathrm{Mn}$ provides direct evidence that there exists strong $\mathrm{Ni}-\mathrm{Ni}$ and $\mathrm{Tb}-\mathrm{Ni}$ exchange interactions as mentioned in ref. [15].

Figure 2 (a) X-ray absorption spectra of the $\mathrm{Mn} L_{23}(2 p \rightarrow 3 d)$ of $\mathrm{TbNi}_{2} \mathrm{Mn}(\mathrm{T}=20 \mathrm{~K})$ and (b) X-ray magnetic circular dichroism of the $\mathrm{Mn} L_{23}(2 p \rightarrow 3 d)$ of $\mathrm{TbNi}_{2} \mathrm{Mn}(\mathrm{T}=20 \mathrm{~K} ; \mathrm{B}=1 \mathrm{~T})$. The peaks labelled A, B, C, D, E agree well with theoretical calculations as discussed in the text.

For Mn, the present XAS and XMCD spectra (Figure 2) agree qualitatively with the atomic calculation based on the transition of $3 d^{5} \rightarrow 2 p 3 d^{6}$ of $\mathrm{Mn}^{2+} \cdot 23$ The observed triple peak structures at the $\mathrm{L}_{3}$ edge and double peak features at the $\mathrm{L}_{2}$ edge of $\mathrm{Mn}$ are consistent with the calculations. Our experimentally measured isotropic branching ratio of $\mathrm{B}(\mathrm{i})^{\text {meas }}=0.87$ is approximately $10 \%$ larger than the calculated values of $\mathrm{B}(\mathrm{i})=0.76$ for the high-spin ground state. ${ }^{23}$ Similar experimental XAS and XMCD spectra for Mn have also been reported from $\mathrm{Co}_{2} \mathrm{MnGe}$ Heusler thin films. ${ }^{28,29}$ Even though x-ray magnetic circular dichroism has been observed for Mn, the derived magnetic moments through the sum rule are in the order of $0.08 \mu_{\mathrm{B}}$ and $0.01 \mu_{\mathrm{B}}$ for spin and orbital part, respectively 
(the spin magnetic moment has been multiplied by a correction factor of $1 / 0.68$ due to strong overlap between $\mathrm{L}_{3}$ and $\mathrm{L}_{2}$ edges ${ }^{28}$ ). As discussed further below, the surprising low magnetic moment for Mn suggests that Mn may not be ferromagnetically ordered in the sample.

Figure 3 (a) X-ray absorption spectra of the $\mathrm{Tb} M_{4,5}\left(4 f \rightarrow 5 d\right.$ ) of $\mathrm{TbNi}_{2} \mathrm{Mn}(\mathrm{T}=20 \mathrm{~K})$ and (b) X-ray magnetic circular dichroism of the $\mathrm{Tb} M_{4,5}(4 f \rightarrow 5 d)$ of $\mathrm{TbNi}_{2} \mathrm{Mn}(\mathrm{T}=20 \mathrm{~K} ; \mathrm{B}=1 \mathrm{~T})$. The peaks labelled A, B, C, D, E correspond well with theoretical calculations as discussed in the text.

The XAS and XMCD spectra for Tb as shown in Figure 3, agree well with the full multiplet calculations in intermediate coupling using the t-matrix approach for the $\mathrm{Tb} 3 d \rightarrow 4 f$ transition [30]. The observed multi-structures of A, B, C, D, and E in the XAS correspond well to the calculated multiplet structures from the strong core-valence exchange interactions. ${ }^{30}$ Due to limited energy resolution, the calculated double features around the peak B are not resolved in the present experiment. Applying the sum rule for the $\mathrm{M}_{4,5}$ edges from $3 d \rightarrow 4 f$ transitions, leads to spin and orbital moments for the $\mathrm{Tb}$ atoms of $0.33 \mu_{\mathrm{B}}$ and $0.19 \mu_{\mathrm{B}}$ respectively. These $\mathrm{Tb}$ moment values have been obtained with an L/S ratio of 0.58 which is very similar to the value of 0.54 from XMCD measurements on Tb-doped $\mathrm{Ni}_{81} \mathrm{Fe}_{19} \cdot{ }^{31}$

Figure 4 (Colour on-line) X-ray magnetic circular dichroism of the $\mathrm{Ni} L_{23}(2 p \rightarrow 3 d)$ of $\mathrm{TbNi}_{2} \mathrm{Mn}$ at $\mathrm{T}=300 \mathrm{~K}$ (red circle and solid line); $\mathrm{T}=80 \mathrm{~K}$ (blue dashed line); $\mathrm{T}=20 \mathrm{~K}$ (black solid line) in an applied magnetic field $\mathrm{B}=1 \mathrm{~T}$.

The XMCD spectra for $\mathrm{Ni}, \mathrm{Mn}$ and $\mathrm{Tb}$ in the $\mathrm{TbNi}_{2} \mathrm{Mn}$ compound have been measured at 300 $\mathrm{K}, 80 \mathrm{~K}$ and $20 \mathrm{~K}$; these temperatures were chosen following the temperature dependent magnetization studies which indicate a magnetic transition temperature of $\mathrm{T}_{\mathrm{C}} \sim 131 \mathrm{~K} .{ }^{13}$ Figure 4 shows the XMCD spectra of Ni, Mn and Tb at these three temperatures (as an aid to comparison the XMCD spectra have been normalised to the isotropic XAS). Despite the low signals from the Mn 
and $\mathrm{Tb}$ elements (Figures 4(b) and 4(c)) compared with the Ni signals (Figure 4(a)), it is evident that the temperature dependence of the XMCD spectra of $\mathrm{Ni}, \mathrm{Mn}$ and $\mathrm{Tb}$ are consistent with a magnetic transition temperature of $\mathrm{T}_{\mathrm{C}} \sim 131 \mathrm{~K}$. The derived magnetic moments are listed in Table I for the three elements. The XMCD signal observed at room temperature is reduced significantly compared with the spectra at low temperatures for all three elements. Considering the error bar of $0.01 \mu_{\mathrm{B}}$, no magnetic moment has been detected at room temperature, apart from the small value of Ni spin moment at this temperature (Table I).

Pronounced and similar XMCD signals have been observed for Ni at $80 \mathrm{~K}$ and $20 \mathrm{~K}$ (Figure 4(a)) at which temperature $\mathrm{Ni}$ almost reaches its maximum magnetic moment. This means that a magnetic field of $1 \mathrm{~T}$ is sufficient to totally align the Ni moment.

As indicated by Figure 4(b), very small XMCD signals were detected for Mn at both $80 \mathrm{~K}$ and $20 \mathrm{~K}$. These small signals lead to a Mn magnetic moment in the order of $<0.1 \mu_{\mathrm{B}}$ as shown in Table I. Such a moment is surprisingly small for a ferromagnetic state; this result suggests that the $\mathrm{Mn}$ magnetic moment may be antiparallel across the 8a and 16d sites for which approximately equal occupancy has been found through x-ray diffraction. ${ }^{13}$ If $\mathrm{Mn}$ atoms at these two sites are ordered in opposite directions, then XMCD will only be able to detect the small amount of net moment even though a moment as large as $1.4 \mu_{\mathrm{B}}$ has been reported by neutron diffraction ${ }^{14}$ which is, of course, used to determine the full magnetic moment from the antiferromagnetic state. Antiparallel magnetic coupling of Mn across the two sublattices $(\mathrm{z}=1 / 8,5 / 8$ and $3 / 8,7 / 8$ ) at the $16 \mathrm{~d}$ sites has been observed in $\mathrm{TbMn}_{2}$ by neutron diffraction. ${ }^{32}$

As reflected by the XMCD spectra for Tb (Figure 4(c)), the measured magnetic moment is found to increase on decreasing the sample temperature from room temperature to $20 \mathrm{~K}$ (Table I). However, even at $20 \mathrm{~K}$, the detected magnetic moment for $\mathrm{Tb}$ is only about $6 \%$ of the maximum saturated value of $9.0 \mu_{\mathrm{B}}$ /atom. The present XMCD spectra indicate that the Tb magnetic moment is aligned in the same direction as that of $\mathrm{Ni}$ as evidenced by the net negative area at the $\mathrm{M}_{5}$ edge in 
the XMCD spectra (Figure 3). This behaviour appears contradictory to the general picture of antiparallel aligned magnetic moment of $\mathrm{Tb}$ and $\mathrm{Ni}$ as supported by neutron diffraction on $\mathrm{TbNi}_{2} \mathrm{Mn}^{14}$ and XMCD observations on $\mathrm{GdNi}_{2} \cdot{ }^{11}$ However it should be noted that Chaboy et al. ${ }^{33}$ demonstrated that XMCD spectra may result in the wrong sign - and hence the orientation of the magnetic moment with respect to the magnetisation direction - for some rare earths such as Gd, Tb, Er and Nd. As outlined by Chaboy et al. $^{33}$, this incorrect identification of the moment direction occurs when the $5 d$ polarization of these rare earths is due to the intra-atomic $4 \mathrm{f}-5 \mathrm{~d}$ exchange, and stems from the spin dependence of the matrix elements previously neglected ${ }^{34}$ and is associated with the radial part of the wave functions for the $5 d$ conduction electrons ${ }^{35}$. It is noted that the question of the alignment between the $\mathrm{Tb}$ and $\mathrm{Ni}$ moments in $\mathrm{TbNi}_{2} \mathrm{Mn}$ should be investigated further. Moreover, considering the strong magnetic fluctuations and non-collinear coupling of $\mathrm{Tb}$ at the 4c site as revealed by the neutron diffraction of $\mathrm{TbNi}_{2} \mathrm{Mn}$ at $4.2 \mathrm{~K},{ }^{14}$ the present observation for the $\mathrm{Tb}$ magnetic moment is likely to occur as a result of a small projection along the external magnetic field. The applied field of $1 \mathrm{~T}$ that can be accessed in our XMCD experiment is far less than the saturation field which is beyond $15 \mathrm{~T}^{14}$ for a spherical sample of $\mathrm{TbNi}_{2} \mathrm{Mn}$ in pulsed magnetic fields (by comparison Wang et al. ${ }^{13}$ obtained a saturation field of $\sim 2 \mathrm{~T}$ for $\mathrm{DC}$ magnetisation measurements on a bulk sample). Similar results have been observed for Tb doped $\mathrm{Ni}_{81} \mathrm{Fe}_{19}$ where only about $2 \%$ of the saturation moment was measured for Tb with XMCD. ${ }^{31}$ Further investigation is required at lower temperatures with scope to apply a much larger magnetic field. This small $\mathrm{Tb}$ moment accounts for the apparent difference between the overall sample magnetic moment resulting from analysis of the present XMCD spectra (about $1.8 \mu_{\mathrm{B}}$ per formula) and the previous results from magnetometer (about $4.8 \mu_{\mathrm{B}}$ per formula). ${ }^{13}$ 
TABLE I. Magnetic moment/atom $\left(\mu_{B}\right)$ values derived from the XMCD sum rule. The error bar is about $0.01 \mu_{B}$, mainly from uncertainty in the light polarization and background subtraction. Considering the error bar, no magnetic moment has been detected at room temperature, apart from the small value of Ni spin moment at this temperature. The ratios of L/S at $300 \mathrm{~K}$ are not shown due to large errors from the very small values of $\mathrm{L}$ and $\mathrm{S}$.

\begin{tabular}{|c|c|c|c|c|c|c|c|c|c|}
\hline & \multicolumn{3}{|c|}{$\mathrm{Ni}$} & \multicolumn{3}{|c|}{$\overline{\mathrm{Mn}}$} & \multicolumn{3}{|c|}{$\overline{\mathrm{Tb}}$} \\
\hline & $\mathrm{L}$ & $S$ & $\mathrm{~L} / \mathrm{S}$ & $\mathrm{L}$ & $S$ & $\mathrm{~L} / \mathrm{S}$ & $\mathrm{L}$ & $S$ & $\mathrm{~L} / \mathrm{S}$ \\
\hline $20 \mathrm{~K}$ & 0.05 & $\begin{array}{l}0.53 \\
\end{array}$ & 0.09 & 0.01 & 0.08 & $\begin{array}{l}0.13 \\
\end{array}$ & 0.19 & 0.33 & 0.58 \\
\hline $80 \mathrm{~K}$ & 0.03 & 0.48 & 0.06 & 0.02 & 0.05 & 0.40 & 0.05 & 0.12 & 0.42 \\
\hline $300 \mathrm{~K}$ & 0.002 & 0.15 & & 0.003 & 0.009 & & 0.002 & 0.004 & \\
\hline
\end{tabular}

\section{Summary}

Significant values of $\mathrm{Ni}$ magnetic moments in the intermetallic compound $\mathrm{TbNi}_{2} \mathrm{Mn}$ of the $\mathrm{MgCu}_{2}$-type structure have been observed directly by $\mathrm{x}$-ray magnetic circular dichroism below the magnetic transition temperature, $T_{C}=131 \mathrm{~K}$. The derived values of $\mathrm{Ni}$ spin and orbital magnetic moments of $0.53 \pm 0.01 \mu_{\mathrm{B}}$ and $0.05 \pm 0.01 \mu_{\mathrm{B}}$ respectively at $20 \mathrm{~K}$, agree well with the results obtained for Ni single crystal of $0.52 \mu_{\mathrm{B}}$ and $0.05 \mu_{\mathrm{B}}{ }^{27}$ indicating that $\mathrm{Ni}$ in $\mathrm{TbNi}_{2} \mathrm{Mn}$ carries an intrinsic magnetic moment that is determined by the localised $3 d$ electrons. The existence of a magnetic moment from Ni indicates that R-T and $\mathrm{T}-\mathrm{T}$ interactions cannot be neglected. This provides clear evidence in support of the mechanism of the enhancement of transition temperature due to the R-T and T-T interactions. The very small magnetic moment from Mn (of order $0.08 \mu_{\mathrm{B}}$ and $0.01 \mu_{\mathrm{B}}$ for the spin and orbital parts) implies that the Mn moment are ordered in opposite directions across the $8 \mathrm{a}$ and $16 \mathrm{~d}$ sites, resulting in the small net moment detected in XMCD measurements. The small Tb magnetic moment (of spin and orbital moment components $0.33 \mu_{\mathrm{B}}$ and $0.19 \mu_{\mathrm{B}}$ respectively) determined from the $20 \mathrm{~K}$ XMCD spectra is considered to be a measure of the moment as projected along the direction of the applied field. 


\section{Acknowledgements}

The authors would like to acknowledge the National Synchrotron Radiation Research Center, Taiwan for access to the BL11A Dragon beam line for the measurements.

\section{References}

${ }^{1}$ K. A. Gschneidner Jr, V. K. Pecharsky and A. O. Tsokol, Rep. Prog. Phys. 68, 1479 (2005).

${ }^{2}$ P. Kumar, K. G. Suresh, A. K. Nigam, A. Magnus, A. A. Coelho and S. Gama, Phys. Rev. B 77, 224427 (2008).

${ }^{3}$ S. Gama, A. A. Coelho, A. de Campos, A. M. Carvalho, F. C. G. Gandra, P. J. von Ranke and N. de Oliveira, Phys. Rev. Lett. 93, 237202 (2004).

${ }^{4}$ V. K. Pecharsky, K. A. Gschneidner Jr , Int. J. Refrig. 29, 1239 (2006).

${ }^{5}$ E. Brück, Handbook of Magnetic Materials , Ed: Buschow K H J, (Elsevier, Amsterdam, The Netherlands) Vol. 17, p235 (2008).

${ }^{6}$ A. Smith, C. R. H. Bahl, R. Bjørk, K. Engelbrecht, K. K. Nielsen, and N. Pryds, Adv. Energy Mater. 2, 1288 (2012).

${ }^{7}$ G. Engdahl, Handbook of Giant Magnetostrictive Materials (Academic Press, New York) (2000).

${ }^{8}$ K. N. R. Taylor, Adv. Phys. 20, 603 (1971).

${ }^{9}$ W. E. Wallace, Rare Earth Intermetallics, (Academic Press, New York), p. 112 (1973).

${ }^{10}$ K. H. J. Buschow, Ferromagnetic Materials, edited by E. P. Wohlfarth, (North-Holland, Amsterdam), Vol. 1, p 297 (1980).

11 M. Mizumaki, K. Yano, I. Umehara, F. Ishikawa, K. Sato, A. Koizumi, N. Sakai and T. Muro, Phys. Rev. B 67, 132404 (2003).

12 K. Yano, Y. Tanaka, I. Matsumoto, I. Umehara, K. Sato, H. Adachi and H. Kawata, J. Phys.: Condens. Matter 18, 6891 (2006).

13 J. L. Wang, C. Marquina, M. R. Ibarra and G. H. Wu, Phys. Rev.B 73, 094436 (2006). 
14 N.V. Mushnikov, V. S. Gaviko, J. Park, and A. N. Pirogov, Phys. Rev. B 79, 184419 (2009).

15 J. L.Wang, S. J. Campbell, S. J. Kennedy, R. Zeng, S. X. Dou and G. H. Wu, J. Phys.: Condens. Matter 23, 216002 (2011).

16 C. T. Chen, Nucl. Instrum. Methods Phys. Res. Sect. A 256, 595 (1987); C. T. Chen and F. Sette, Rev. Sci. Instrum., 60, 1616, (1989).

17 H. J. Elmers, G. H. Fecher, D. Valdaitsev, S. A. Nepijko, A. Gloskovskii, G. Jakob, G. Schonhense, S. Wurmehl, T. Block, C. Felser, P. C. Hsu and W. L. Tsai, Phys. Rev. B 67, 104412 (2003).

18 B. T. Thole, P. Carra, F. Sette, and G. van der Laan, Phys. Rev.Lett. 68, 1943 (1992).

19 P. Carra, B. T. Thole, M. Altarelli, and X. Wang, Phys. Rev. Lett.70, 694 (1993).

20 S. Asano and I. Ishida, Solid State Phys. 21, 711 (1986).

21 I. Galanakis, P. H. Dederichs, and N. Papanikolaou, Phys. Rev. B66, 174429 (2002).

22 Y. Teramura, A. Tanaka, B. Thole, and T. Jo, J. Phys. Soc. Jpn. 65, 3056 (1996).

23 G. van der Lann, and B. T. Thole, Phys. Rev. B 43, 13401 (1991).

24 G. van der Lann, C. M. B. Henderson, R. A. D. Pattrick, S. S. Dhesi, P. F. Schofield, E. Dudzik, and D. J. Vaughan, Phys. Rev. B 59, 4314 (1999).

D. H. Yu, J. Duriavig, N. Loh, R. Woodward, H. J. Lin, F. H. Chang, S. H. Kilcoyne, A. Stampfl, and R. A. Robinson, Appl. Phys. Lett. 94, 022502 (2009). 4253 (1986).

27 C. T. Chen, N. V. Smith, and F. Sette, Phys. Rev. B 43, 6785 (1991).

28 J. Grabis, A. Bergmann, A. Nefedov, K. Westerholt, and H. Zabel, Phys. Rev. B 72, 024437 (2005). 
V. R. Singh, V. K. Verma, K. Ishigami, G. Shibata, T. Kadono, A. Fujimori, D. Asakura, T. Koide, Y. Miura, M. Shirai, G. F. Li, T. Taira and M. Yamamoto, Phys. Rev. B 86, 144412 (2012).

${ }^{30}$ G. van der Lann, E. Arenholz, Z. Hu, A. Bauer, E. Weschke, Ch. Schüssler-Langeheine, E. Navas, A. Mühlig, G. Kaindl, J. B. Goedkoop and N. B. Brookes, Phys. Rev. B 59, 8835 (1999). 31 Y. Guan, Z. Dios, D. A. Arena, L. Cheng and W. E. Bailey, J. Appl. Phys. 97, 10A719 (2005).

32 L. M. Corliss and J. M. Hastings, J. Appl. Phys. 35, 1051 (1964).

33 J. Chaboy, M. A. Laguna-Marco, C. Piquer, H. Maruyama and N. Kawamura, J. Phys.: Condens. Matter 19, 436225 (2007).

${ }^{34}$ X. Wang, T. C. Leung, B. N. Harmon and P. Carra, Phys. Rev. B 47, 9087 (1993).

35 B. N. Harmon and A. J. Freeman, Phys. Rev. B 10, 1979 (1974). 


\section{Figure Captions}

Figure 1 (a) X-ray absorption spectra of the $\mathrm{Ni} L_{23}(2 p \rightarrow 3 d)$ of $\mathrm{TbNi}_{2} \mathrm{Mn}(\mathrm{T}=20 \mathrm{~K})$ and

(b) X-ray magnetic circular dichroism of the $\mathrm{Ni} L_{23}(2 p \rightarrow 3 d)$ of $\mathrm{TbNi}_{2} \mathrm{Mn}(\mathrm{T}=20 \mathrm{~K}$; $\mathrm{B}=1 \mathrm{~T})$.

The peaks labelled A, B, C, D, E agree well with theoretical calculations as discussed in the text.

Figure 2 (a) X-ray absorption spectra of the $\mathrm{Mn} L_{23}(2 p \rightarrow 3 d)$ of $\mathrm{TbNi}_{2} \mathrm{Mn}(\mathrm{T}=20 \mathrm{~K})$ and

(b) X-ray magnetic circular dichroism of the $\mathrm{Mn} L_{23}(2 p \rightarrow 3 d)$ of $\mathrm{TbNi}_{2} \mathrm{Mn}(\mathrm{T}=20 \mathrm{~K} ; \mathrm{B}=1 \mathrm{~T})$.

The peaks labelled A, B, C, D, E agree well with theoretical calculations as discussed in the text.

Figure 3 (a) X-ray absorption spectra of the $\mathrm{Tb} M_{4,5}(4 f \rightarrow 5 d)$ of $\mathrm{TbNi}_{2} \mathrm{Mn}(\mathrm{T}=20 \mathrm{~K})$ and

(b) X-ray magnetic circular dichroism of the $\mathrm{Tb} M_{4,5}(4 f \rightarrow 5 d)$ of $\mathrm{TbNi}_{2} \mathrm{Mn}(\mathrm{T}=20 \mathrm{~K} ; \mathrm{B}=1 \mathrm{~T})$.

The peaks labelled A, B, C, D, E correspond well with theoretical calculations as discussed in the text.

Figure 4 (Colour on-line) X-ray magnetic circular dichroism of $\mathrm{TbNi}_{2} \mathrm{Mn}$ at $\mathrm{T}=300 \mathrm{~K}$ (red circle and solid line); $\mathrm{T}=80 \mathrm{~K}$ (blue dashed line); $\mathrm{T}=20 \mathrm{~K}$ (black solid line) in an applied magnetic field $\mathrm{B}=1 \mathrm{~T}$

(a) Ni $L_{23}(2 p \rightarrow 3 d)$;

(b) $\operatorname{Mn} L_{23}(2 p \rightarrow 3 d)$;

(c) $\mathrm{Tb} M_{4,5}(4 f \rightarrow 5 d)$. 


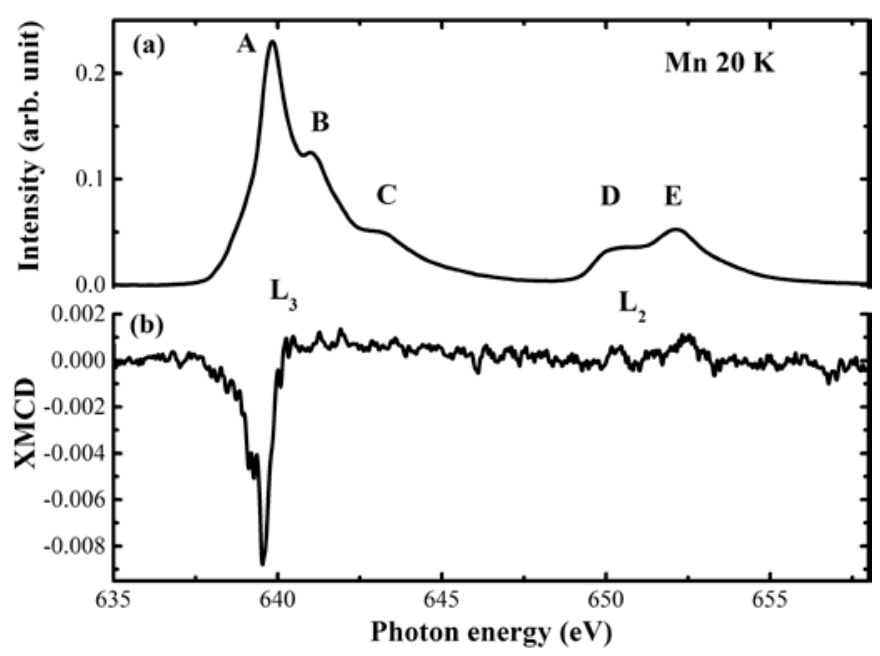

Figure 1 (a) X-ray absorption spectra of the $\mathrm{Ni} L_{23}(2 p \rightarrow 3 d)$ of $\mathrm{TbNi}_{2} \mathrm{Mn}(\mathrm{T}=20 \mathrm{~K})$ and

(b) X-ray magnetic circular dichroism of the $\mathrm{Ni} L_{23}(2 p \rightarrow 3 d)$ of $\mathrm{TbNi}_{2} \mathrm{Mn}(\mathrm{T}=20 \mathrm{~K}$; $\mathrm{B}=1 \mathrm{~T})$.

The peaks labelled A, B, C, D, E agree well with theoretical calculations as discussed in the text.

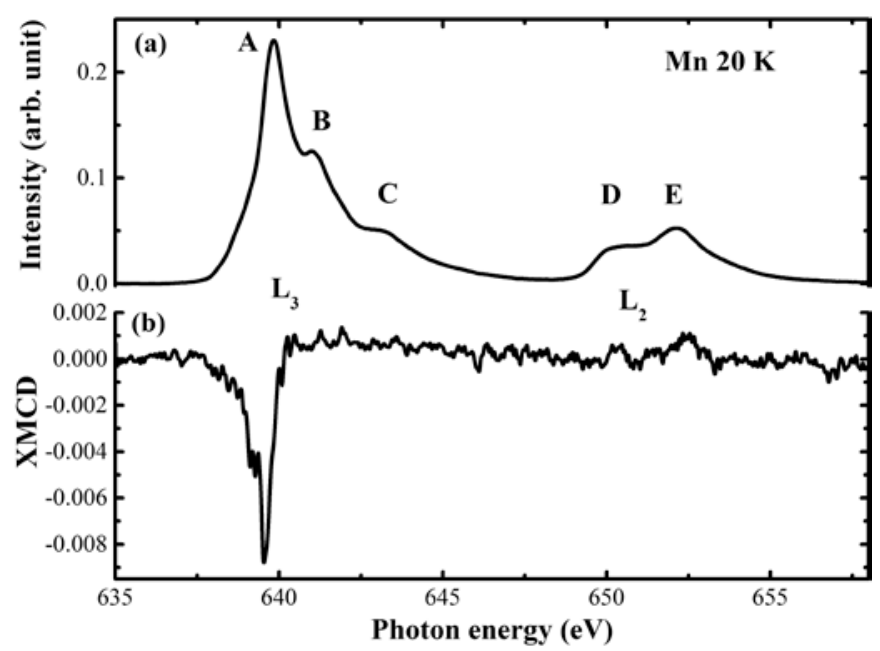

Figure 2 (a) X-ray absorption spectra of the $\mathrm{Mn} L_{23}(2 p \rightarrow 3 d)$ of $\mathrm{TbNi}_{2} \mathrm{Mn}(\mathrm{T}=20 \mathrm{~K})$ and (b) X-ray magnetic circular dichroism of the $\mathrm{Mn} L_{23}(2 p \rightarrow 3 d)$ of $\mathrm{TbNi}_{2} \mathrm{Mn}(\mathrm{T}=20 \mathrm{~K}$; $\mathrm{B}=1 \mathrm{~T})$. The peaks labelled A, B, C, D, E agree well with theoretical calculations as discussed in the text. 


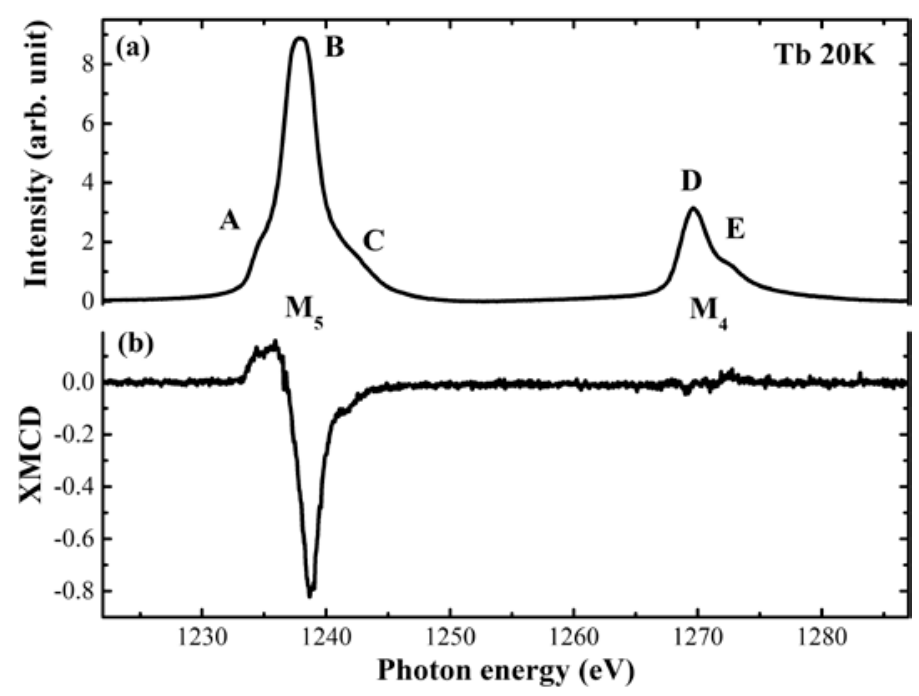

Figure 3 (a) X-ray absorption spectra of the $\mathrm{Tb} M_{4,5}(4 f \rightarrow 5 d)$ of $\mathrm{TbNi}_{2} \mathrm{Mn}(\mathrm{T}=20 \mathrm{~K})$ and (b) X-ray magnetic circular dichroism of the $\mathrm{Tb} M_{4,5}(4 f \rightarrow 5 d)$ of $\mathrm{TbNi}_{2} \mathrm{Mn}(\mathrm{T}=20 \mathrm{~K}$; $\mathrm{B}=1 \mathrm{~T})$. The peaks labelled A, B, C, D, E correspond well with theoretical calculations as discussed in the text. 
(a)

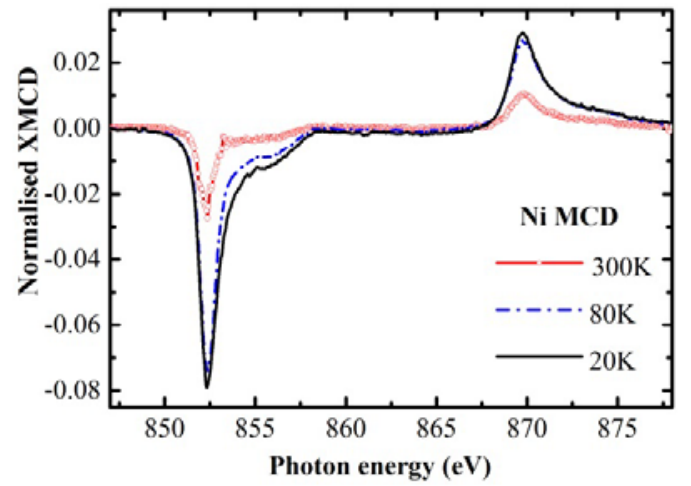

(b)

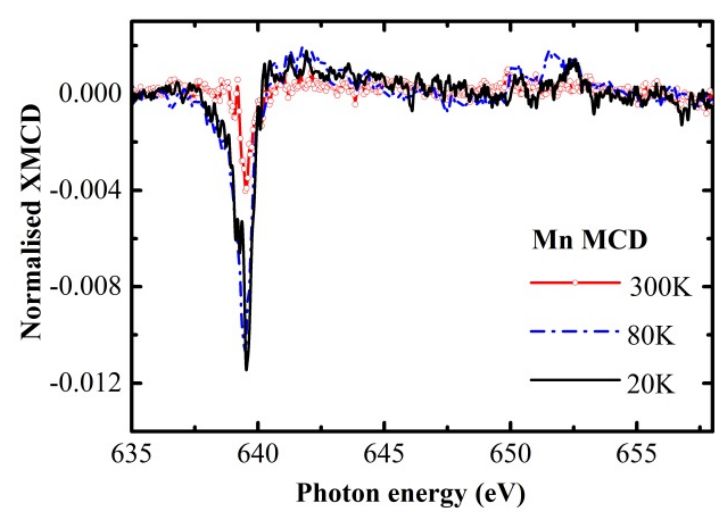

(c)

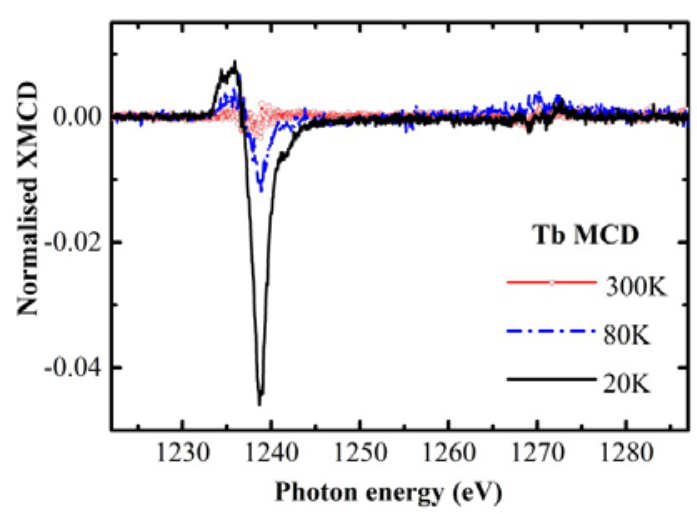

Figure 4 (Colour on-line) X-ray magnetic circular dichroism of $\mathrm{TbNi}_{2} \mathrm{Mn}$ at $\mathrm{T}=300 \mathrm{~K}$ (red circle and solid line); $\mathrm{T}=80 \mathrm{~K}$ (blue dashed line); $\mathrm{T}=20 \mathrm{~K}$ (black solid line) in an applied magnetic field $\mathrm{B}=1 \mathrm{~T}$

(a) Ni $L_{23}(2 p \rightarrow 3 d)$;

(b) $\mathrm{Mn} L_{23}(2 p \rightarrow 3 d)$;

(c) $\mathrm{Tb} M_{4,5}(4 f \rightarrow 5 d)$. 